\title{
Lesson of the month 2: Transient reversible amnesia in multiple sclerosis
}

\author{
Authors: Priya Devi Shanmugarajah, ${ }^{A}$ Jane Alty, ${ }^{B}$ Oliver Lily ${ }^{B}$ and Helen L Ford ${ }^{B}$
}

Transient amnestic syndromes are fascinating clinical entities and there are several subtypes. Transient global amnesia (TGA) is characterised by sudden onset of anterograde amnesia with repetitive questioning, lasting less than 24 hours. The pathophysiology of TGA involves the medial temporal lobes and hippocampi. Episodes of TGA are thought to involve venous congestion with Valsalvalike activities, vascular or migrainous mechanisms. In contrast, transient epileptic amnesia manifests as brief and frequent episodes of amnesia due to seizure activity in the temporal lobes. Transient memory disturbances can also be caused by transient ischaemic attack. We describe the first reported case of transient reversible amnesia directly attributable to acute demyelination. This case reminds us that multiple sclerosis relapses may present with acute cognitive impairment rather than the more classical physical symptoms. This is an important learning point in terms of appropriate management and eligibility for disease-modifying drugs.

KEYWORDS: Anterograde amnesia, demyelinating disorders, multiple sclerosis, temporal lobe

\section{Introduction}

Transient amnestic syndromes are fascinating clinical entities and there are several subtypes. Transient global amnesia (TGA) is characterised by sudden onset of an inability to form new memories (known as anterograde amnesia), accompanied by repetitive questioning. ${ }^{1}$ Patients remain fully conscious, often carrying out complex tasks like driving and other cognitive domain remain intact. ${ }^{1,2}$ An episode of TGA lasts less than 24 hours - usually between 1 and 8 hours. ${ }^{2}$ The hippocampus and medial temporal lobe are involved in the formation and retrieval of new episodic memory and these areas are thought to

Authors: A specialist registrar in neurology, Department of Neurology, Leeds Centre for Neurosciences, Leeds Teaching

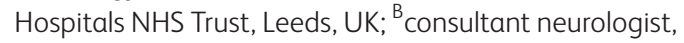
Department of Neurology, Leeds Centre for Neurosciences, Leeds Teaching Hospitals NHS Trust, Leeds, UK be affected in TGA. ${ }^{1,2}$ The majority of TGA cases are thought to be due to one of the following mechanisms:

$>$ a vascular aetiology secondary to arterial ischemia or venous congestion with Valsalva manoeuvre

$>$ cortical spreading depression associated with migrainous phenomena $^{1,2}$

> altered neuronal excitability related to an epileptic seizure. ${ }^{3}$

Transient epileptic amnesia (TEA) is often mistaken for TGA but TEA episodes are briefer and more frequent. They typically last less than 1 hour and are often accompanied by other features associated with epilepsy, such as oroalimentary automatism and brief unresponsive episodes. ${ }^{2,3}$ Isolated transient memory impairment secondary to posterior circulation transient ischaemic attacks (TIA) are usually associated with other signs, such as ataxia, dysarthria or nystagmus. $^{2}$

We describe the first reported case of transient reversible amnestic syndrome secondary to acute demyelination that led to a new diagnosis of multiple sclerosis (MS).

\section{Case history}

A 29-year-old right-handed man presented with acute onset of memory impairment. His girlfriend explained that a week earlier, he suddenly became lost while driving his usual route home from work. He telephoned her to ask for help and sounded upset and agitated. She managed to locate him by directing him to read out nearby road signs but when she arrived to pick him up, he had already driven off, having forgotten that he had called her. After locating him, he repeatedly asked her the same questions and could not lay down any new memories. He recovered significantly within the first 24 hours but had remained somewhat forgetful since then. There was no history of associated headache, fever, seizure activity, motor or sensory impairment or precipitating psychosocial stressors.

He had presented 4 months earlier with a week-long history of progressive ascending paraesthesia up to his clavicles and fingertips. Examination had revealed a sensory level at C6. Magnetic resonance imaging (MRI) of his brain and cervical spine had shown widespread T2 hyperintensity in the brain and cervical cord compatible with demyelination. Routine blood tests were normal. Paired oligoclonal bands were positive in the cerebrospinal fluid only. He was diagnosed with clinically 


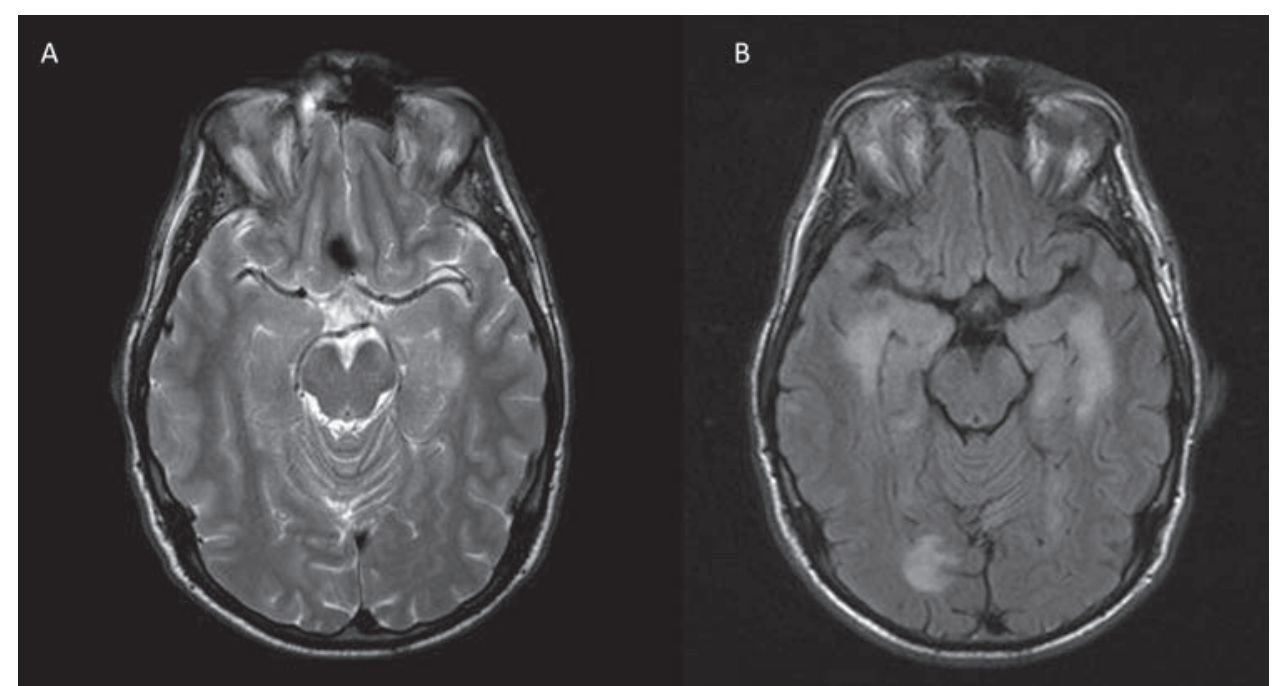

Fig 1. Magnetic resonance imaging (MRI) scans of patient. A - MRI scan 4 months prior to the acute episode of amnesia. Axial MRI T2-weighted image shows subtle signal change lateral to temporal horn (left>right) with concomitant juxto-cortical signal change in the right occipital lobe. B - MRI scan 2 weeks after the onset of episode of amnesia. Axial MRI FLAIR sequence showed new areas of $\mathrm{T} 2$ high signal consistent with demyelination that predominantly affected both temporal lobes. isolated syndrome (CIS) and treated with a 3-day course of intravenous methylprednisolone. The patient made a good recovery with mild residual fatigue and paraesthesiae in the hands. CIS is a single clinical demyelinating event of the central nervous system; it is usually the first event of MS although the time to second event can be variable.

On examination, his short-term memory was severely disrupted, forming virtually no new memories with repeated questioning. His speech was normal in form and content. He scored 22/30 on the mini mental state examination (MMSE), losing points for serial 7s, delayed recall and orientation in time. The lower limb reflexes were pathologically brisk but the rest of the neurological examination was normal.

He was diagnosed with an isolated transient amnestic syndrome due to a second demyelinating episode. Repeat MRI of the brain showed new areas of T2 hyperintensity consistent with demyelination that predominantly affected both temporal lobes (Fig 1). He was managed conservatively and the memory disturbance completely resolved over a few weeks. Repeat MMSE testing 3 weeks later was normal.

As the transient reversible amnestic syndrome was considered to be a second demyelinating episode, separated in space and time, he was thus diagnosed with relapsing remitting multiple sclerosis (RRMS). In view of two significant clinical events within 4 months, he was eligible for disease-modifying drugs and was commenced on beta-interferon treatment. Over a 6-year follow-up period, he has had no further new symptoms.

\section{Discussion}

It is rare for an acute relapse to present with an isolated episode of amnesia. To the best of the authors' knowledge, this is the first reported case of an isolated transient reversible amnestic syndrome secondary to demyelination. The only other similar case previously reported was by Jenkins et al, ${ }^{3}$ who described a case of MS presenting with recurrent brief amnestic episodes; however, these were postulated to be due to a TEA. The seizure activity was thought to be precipitated by the underlying inflammation in the temporal lobes. ${ }^{3}$
Our patient presented with an isolated acute episode of anterograde amnesia with repeated questioning. The clinical features of this case meet the diagnostic criteria for TGA, except the patient's impaired attention and duration of symptoms lasted several weeks. TGA is classically defined as lasting less than 24 hours with other cognitive domain remaining intact. This patient's isolated reversible amnestic episode lasted a few weeks, suggesting that the new inflammatory lesions seen in the temporal lobes on the repeat MRI contributed to the prolonged duration of the reversible amnestic episode. Limbic encephalitis antibody testing was not performed in this case as the memory impairment was self-limiting without treatment and he did not have any other clinical signs of encephalitis.

It was important to recognise that this patient's transient amnestic syndrome was a second episode of demyelination separated in space and time. This changed his diagnosis from CIS to RRMS. He also became eligible for disease-modifying drugs, which are only prescribed to patients with two significant relapses within 2 years.

\section{Conclusion}

It is important for clinicians to recognise that MS relapses can present solely with an acute onset of reversible amnesia rather than the more classical signs of optic neuritis and paraparesis. ${ }^{3}$ This is the first case reported of RRMS presenting with the symptoms of transient reversible amnesia with the likely aetiology postulated to the inflammatory demyelinating lesions in the medial temporal along with other cortical lesions. Early recognition led to initiation of disease-modifying treatment and he has remained relapse-free since this episode.

\section{Key learning points}

> It is important for clinicians to recognise that multiple sclerosis can present solely with an acute onset of amnesia, or indeed with other cognitive and psychiatric symptoms.

$>$ Transient global amnesia affects anterograde memory resulting in repetitive questioning. 
> Transient epileptic amnesia presents as brief and frequent periods of amnesia, usually with clouding of consciousness.

> Posterior circulation transient ischaemic attacks may also present with reversible memory impairment often associated with brainstem or cerebellar signs.

$>$ This is the first case reported of transient reversible amnesia secondary to acute demyelination that led to a new diagnosis of multiple sclerosis

\section{Conflicts of interest}

The authors have no conflicts of interest to declare.

\section{Acknowledgements}

Written consent was obtained from the patient to publish the clinical details and images in this article.

The authors would like to thank Dr Daniel Warren, consultant neuroradiologist at Leeds Teaching Hospitals NHS Trust, for providing the images and figure legends for this paper and also Dr Anna Kilsby, consultant neuropsychiatrist at Leeds and York Partnership NHS Foundation Trust, for her psychiatric assessment of the patient.

\section{References}

1 Owen D, Paranandi B, Sivakumar B, Seevaratnam M. Classical diseases revisited: transient global amnesia. Postgrad Med J 2007;83:236-9.

2 Butler CR, Zeman AZJ. Syndromes of transient amnesia. Adv Clin Neurosci Rehabil 2006;6:13-4.

3 Jenkins PO, Perry R, Malik M. Multiple sclerosis presenting as a relapsing amnestic syndrome. Pract Neurol 2014;14:100-1.

Address for correspondence: Dr Priya Devi Shanmugarajah, Neurology Department, Leeds General Infirmary, Great George Street, Leeds LS1 3EX, UK.

Email: pshanmugarajah@doctors.org.uk 